\title{
All-optical suppression of relativistic self-focusing of laser beams in plasmas
}

\author{
S. Yu. Kalmykov, ${ }^{*}$ S. Austin Yi, and G. Shvets \\ The Department of Physics and Institute for Fusion Studies, The University of Texas at Austin, One University Station C1500, \\ Austin, Texas 78712, USA
}

(Received 1 April 2008; revised manuscript received 19 October 2008; published 21 November 2008)

\begin{abstract}
It is demonstrated that a catastrophic relativistic self-focusing (RSF) of a high-power laser pulse can be prevented all-optically by a second, much weaker, copropagating pulse. RSF suppression occurs when the difference frequency of the pulses slightly exceeds the electron plasma frequency. The mutual defocusing is caused by the three-dimensional electron density perturbation driven by the laser beat wave slightly above the plasma resonance. A bienvelope model describing the early stage of the mutual defocusing is derived and analyzed. Later stages, characterized by the presence of a strong electromagnetic cascade, are investigated numerically. Stable propagation of the laser pulse with weakly varying spot size and peak amplitude over several Rayleigh lengths is predicted.
\end{abstract}

DOI: 10.1103/PhysRevE.78.057401

PACS number(s): $52.38 . \mathrm{Hb}, 52.35 . \mathrm{Mw}, 52.38 . \mathrm{Bv}$

Self-focusing of a high-power laser beam is common to a variety of media: From solid optical fibers to gaseous plasmas [1]. It is caused by the nonlinear dependence of the refractive index on the radiation intensity. In plasmas [2-7], the nonlinear index variation may occur due to the relativistic mass increase of an electron oscillating in the laser field. If the laser power exceeds the threshold $P_{c r}$ $=16.2\left(\omega_{0} / \omega_{p e}\right)^{2} \mathrm{GW}$ [4] [where $\omega_{0}$ is the laser frequency, $\omega_{p e}=\left(4 \pi e^{2} n_{0} / m_{e}\right)^{1 / 2} \ll \omega_{0}$ is the electron plasma frequency, $n_{0},-e$, and $m$ are, respectively, the electron density, charge, and rest mass], relativistic self-focusing (RSF) overcomes diffraction and causes the beam to collapse. Electron cavitation $[4,6]$ or higher-order relativistic nonlinearities $[5,7]$ saturate the RSF.

The laser pulse collapse is undesirable for many plasma based applications (e.g., laser driven acceleration, radiation generation) where stable propagation over many Rayleigh lengths is preferred. In this Brief Report we explore an alloptical technique of suppressing the RSF of a long pulse $\left(\omega_{p e} \tau_{L} \gg 1\right)$ by optical mixing with a second, much weaker pulse shifted in frequency by $\Omega>\omega_{p e}$. The effect of suppression owes to the defocusing properties of the threedimensional (3D) electron density perturbation driven by the ponderomotive force at the difference frequency $\Omega$. Unlike the earlier studied $[8,9]$ reduction of the RSF for ultrashort pulses $\left(\omega_{p e} \tau_{L}<1\right)$, the present effect takes place for long pulses with a large energy.

We theoretically describe the suppression mechanism in terms of a bienvelope propagation model. We assume that two axisymmetric pulses of the same polarization $\mathbf{a}(r, z, \xi)$ $=\operatorname{Re}\left(\mathbf{e}_{0} \Sigma_{l=0,1} a_{l} e^{-i \omega_{l} \xi / c}\right)$ propagate through a uniform rarefied plasma in the positive $z$ direction. Here, $\mathbf{e}_{0}$ is a unit complex vector of polarization, $a_{l}$ are the slowly varying amplitudes of vector potential normalized to $m c^{2} /|e|, r=\left(x^{2}+y^{2}\right)^{1 / 2}$ is the distance from the axis, and $\xi=c t-z$. Ions remain immobile because of the short pulse duration. We assume that the electrons respond instantly to the laser ponderomotive force with a frequency $\Omega=\omega_{0}-\omega_{1} \ll \omega_{0}$, and neglect the effect of radial

*kalmykov@physics.utexas.edu ponderomotive force (the laser waist size $r_{0}$ should thus exceed $\left.k_{p}^{-1}=c / \omega_{p e}\right)$. The electron density perturbation takes the form $\left(n_{e}-n_{0}\right) / n_{0}=\operatorname{Re}\left(N_{e} e^{i \Omega \xi / c}\right)$, where

$$
N_{e}=G_{N_{e}} a_{0}^{*} a_{1} \approx a_{0}^{*} a_{1}\left(\widetilde{\Omega}^{2} / 2\right) /\left(\widetilde{\Omega}^{2}-1\right),
$$

and $\widetilde{\Omega}=\Omega / \omega_{p e}$. Neglecting the electron inertia requires both resonant and relativistic effects in the longitudinal motion to be insignificant, which holds for $|\widetilde{\Omega}-1|$ $>(3 / 4)\left(3\left|a_{0} a_{1}\right|^{2} / 2\right)^{1 / 3} \gg\left(\omega_{p e} \tau_{L}\right)^{-1}[11]$. As we shall see later, the bicolor approximation is violated after a fraction of a Rayleigh length $Z_{R_{0}}=\omega_{0} r_{0}^{2} / 2 c$ because of electromagnetic cascade generation [10-14]. This propagation stage will be studied numerically in particle-in-cell (PIC) simulations via code WAKE [6].

Starting from the Maxwell equations for the laser beams, neglecting the group velocity dispersion of light, using Eq. (1) for the plasma response, and applying a weakly relativistic approximation [i.e., calculating plasma currents with the accuracy $\left.\sim O\left(a^{3}\right)\right]$, we obtain a set of coupled nonlinear paraxial envelope equations,

$$
\begin{gathered}
\left(2 i k_{l} \partial / \partial z+r^{-1} \partial / \partial r+\partial^{2} / \partial r^{2}\right) a_{l} \approx-k_{p}^{2} \chi_{l} a_{l}, \\
\chi_{l}=-\left(\left|a_{l}\right|^{2}+2 G\left|a_{m}\right|^{2}\right) / 4, \\
G=1-G_{N_{e}},
\end{gathered}
$$

where $k_{l}=\omega_{l} / c$, and the subscripts $(l, m)=(0,1)$ and $(1,0)$. The first term in the nonlinear susceptibility (2b) describes the relativistic self-focusing, and the second term describes the nonlinear cross focusing. Their relative magnitude and sign determine whether $\chi_{l}$ leads to the nonlinear focusing or defocusing. The first term in the cross-focusing coefficient (2c) results from the relativistic mass effect $[15,16]$, and the second is the coefficient of plasma wave-induced cross focusing. Its sign is determined by the magnitude of the difference frequency $\widetilde{\Omega}$.

Strong overdetuning $(\widetilde{\Omega} \gg 1)$ makes the plasma response weak but not negligible (and definitely suitable for the electron acceleration [17]). In this case $G \approx 1 / 2$, and the nonlin- 
ear susceptibility [18] $\chi_{0} \approx \chi_{1} \approx-\left(\left|a_{0}\right|^{2}+\left|a_{1}\right|^{2}\right) / 4$ is the same for both pulses. If the pulses entirely overlap at the plasma entrance, the nonlinear refraction is determined by the sum of individual powers (and not by the power partition). This conclusion is supported by the PIC simulations below. In the much more interesting case of $\widetilde{\Omega} \approx 1$ the cross-focusing coefficient is determined almost entirely by the plasma wave contribution, $G \approx[4(1-\widetilde{\Omega})]^{-1}$, hence $|G| \gg 1$. The nonlinear refraction depends now on both power partition and the magnitude of the difference frequency: Nonlinear focusing enhances if $\widetilde{\Omega}<1$, while for $\widetilde{\Omega}>1$ mutual defocusing occurs. The latter case is the main focus of this Brief Report.

We study the dynamics of nonlinear refraction using the source-dependent expansion (SDE), a universal technique earlier applied to free-electron lasers [19], laser-plasma $[5,15,18]$ and electron beam-plasma [20] interactions. Within the SDE framework, each pulse in Eqs. (2) is approximated with the lowest order Gaussian mode, $a_{l}=\left(A_{l} r_{l} / r_{s l}\right) \exp \left[i\left(\theta_{l}\right.\right.$ $\left.\left.+\alpha_{l} r^{2} / r_{s l}^{2}\right)-r^{2} / r_{s l}^{2}\right]$, where $A_{l}(\xi)$ are the beam amplitudes, $r_{s l}(z, \xi)$ are the spot sizes, $r_{l}=r_{s l}(z=0)$ are the spot sizes in the focal plane, and $\alpha_{l}(z, \xi)=\left(k_{l} / 4\right) \partial r_{s l}^{2} / \partial z$. A straightforward calculation yields the equations for the spot size evolution,

$$
\frac{\partial^{2} r_{s l}}{\partial z^{2}}=\frac{4}{k_{l}^{2} r_{s l}^{3}}\left(1-W_{l}-W_{m} \frac{8 G r_{s l}^{4}}{\left(r_{s l}^{2}+r_{s m}^{2}\right)^{2}}\right),
$$

where $W_{l}(\xi)=A_{l}^{2}(\xi) k_{p}^{2} r_{l}^{2} / 32$ is the instant power normalized to the critical power of a monochromatic beam $P_{c r}$ [4]. If the common focal plane of the beams coincides with the plasma entrance, then the boundary conditions are $r_{s l}(0)=r_{l}$, and $\partial r_{s l} / \partial z(0)=0$. Beam slices taken at different $\xi$ focus or defocus independently. This local description is enabled by the local form of plasma response (1), which is itself a consequence of the nonresonant plasma wave excitation. Parameter $G \neq 1$ is responsible for the refractive effect of the plasma wave.

The stationary points of Eqs. (3) correspond to the matched propagation (mutual guiding) of the pulses. The condition for matched propagation gives the normalized instant powers $W_{l}$ in terms of the cross-focusing coefficient $G$ and the area ratio $R=\left(r_{0} / r_{1}\right)^{2}$,

$$
W_{l}=\frac{(1+R)^{2}\left[(1+R)^{2}-8 G R^{2 m}\right]}{\left[(1+R)^{2}-8 G R\right]\left[(1+R)^{2}+8 G R\right]} .
$$

Representative solutions (4) are displayed in Fig. 1(a) for $\widetilde{\Omega}<1$, and in Fig. 1(b) for $\widetilde{\Omega}>1$. In either case, the beam of higher power can guide the lower-power beam with a smaller spot.

The requirement $W_{l}>0$ limits the range of possible $G$ and $R$. When $\widetilde{\Omega}<1$, the matched solutions exist for $D^{-1}<R$ $<D$, where $D \approx 2\left(1-\widetilde{\Omega}^{2}\right)^{-1 / 2}$. Figure 1 (a) shows that the mutual guiding requires less power than the self-guiding of a single beam $\left(W_{0}+W_{1}<1\right)$. Moreover, as $\widetilde{\Omega} \rightarrow 1$, the focusing effect of the plasma wave prevails (the effect dubbed the "cascade focusing" in Refs. [12,13]). For instance, to be matched, the equal-area beams $(R=1)$ with $\widetilde{\Omega}=0.9$ need the
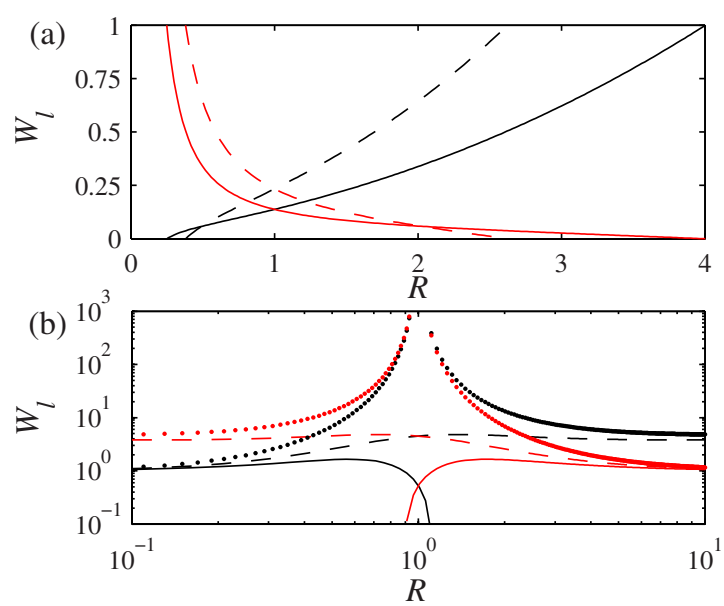

FIG. 1. (Color online) Normalized power for matched solutions $W_{0}$ (black line) and $W_{1}$ [red (dark gray) line $]$ for (a) $\widetilde{\Omega}<1[\widetilde{\Omega}$ $=0.75$ (dashed line), $\widetilde{\Omega}=0.9$ (solid line) $),($ b) $\widetilde{\Omega}>1[\widetilde{\Omega}=\sqrt{3 / 2}($ dot ted line), $\widetilde{\Omega}=1.25$ (dashed line), $\widetilde{\Omega}=3.18$ (solid line)].

power $W_{l} \approx 0.138$, which is less than one-half of that given by the relativistic mass effect alone, $W_{l}(G=1)=1 / 3[15,16]$.

Nonlinear refraction is entirely different in the regimes with overdetuning $(\tilde{\Omega}>1)$. Generally, as shown in Fig. 1(b), to compensate for the plasma wave-induced defocusing, matched beams must have the total power well above $P_{c r}$ (i.e., $\left.W_{0}+W_{1}>1\right)$. Moreover, in the regime with moderate overdetuning $(1<\widetilde{\Omega} \leqslant \sqrt{3 / 2})$, the plasma wave-induced defocusing overcomes the net effect of relativistic self- and cross-focusing, and matching the beams with similar parameters $\left(W_{0} \approx W_{1}, R \approx 1\right)$ becomes impossible. For larger detuning, $\sqrt{3 / 2}<\widetilde{\Omega}<\sqrt{2}$, matching requires an overcritical power for each beam, $W_{l} \approx\left(\widetilde{\Omega}^{2}-1\right) /\left(2 \widetilde{\Omega}^{2}-3\right)>1$. Finally, in the limit of strong overdetuning $(\widetilde{\Omega} \rightarrow \infty)$, only equal-area beams with the total power equal to $P_{c r}$ (i.e., $\left.W_{0}+W_{1}=1\right)$ can be matched. In agreement with the earlier discussion, the condition of mutual guiding is additive in power and independent of the power partition.

Enhanced mutual defocusing of slightly overdetuned beams $(1<\widetilde{\Omega}<\sqrt{2})$ can be used in experimental practice to prevent the catastrophic RSF of a single overcritical laser pulse. The equal-area pulses of different power cannot be mutually guided; therefore, splitting a small fraction of the pulse energy into the frequency-shifted sideband with the same spot size and duration can turn the catastrophic RSF of a monochromatic pulse into a mild defocusing of the bicolor pulse. To this effect, the normalized power of the split pulse must exceed the threshold

$$
W_{1} \geqslant\left(W_{\text {tot }}-1\right)\left(\widetilde{\Omega}^{2}-1\right) .
$$

Here, $W_{\text {tot }}=W_{0}+W_{1}>1$ is the normalized power of the original pulse (the pulse labeled " 0 " can still be overcritical, $W_{0}$ $>1)$.

We support the predictions of the bienvelope model with the PIC simulations using the code WAKE [6]. It solves for the laser beam envelope in the extended paraxial approxima- 


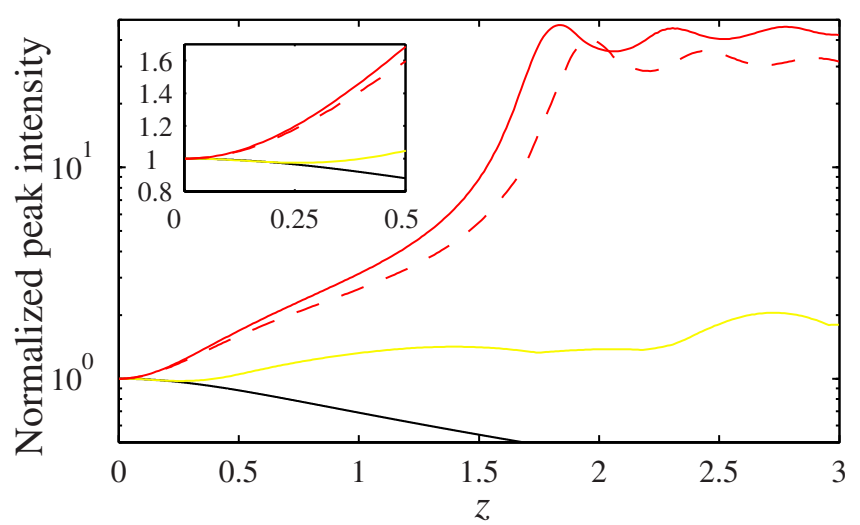

FIG. 2. (Color online) Peak intensity versus propagation distance (in units of $Z_{R_{0}}$ ). The laser is either monochromatic [solid red (dark gray) line] or bicolor with the power partition $W_{0} / W_{1}=17 / 3$ $[\widetilde{\Omega}=3.18$, dashed red (dark gray line); $\widetilde{\Omega}=1.25$, yellow (light gray) and black line]. Black curve is obtained from the bienvelope theory, others are from WAKE simulations. Inset shows the initial stage of evolution.

tion (group velocity dispersion included) in a 3D geometry with cylindrical symmetry. Plasma response to the timeaveraged (over laser period) ponderomotive force is quasistatic. An overcritical bicolor pulse $\left(W_{\text {tot }}=1.1, k_{p} r_{l}=16.5\right.$, $\left.\omega_{p e} \tau_{L} \approx 55, a_{0}^{2}+a_{1}^{2}=0.13\right)$ has been numerically propagated from $z=0$ (focal plane) to $z=3 Z_{R_{0}}$ through a homogeneous preformed plasma with $\omega_{p e} / \omega_{0}=0.018$. For the $0.8 \mu \mathrm{m}$ laser wavelength, these normalized parameters correspond to the $55 \mathrm{TW}$ total power, $\tau_{L}=1.3 \mathrm{ps}$, and plasma length $16 \mathrm{~cm}$.

When the pulse is monochromatic $\left(a_{1}=0\right)$, its central part (where $W_{0} \geqslant 1$ ) undergoes a catastrophic RSF. The peak intensity grows by a factor of $\sim 50$ within two Rayleigh lengths as shown in Figs. 2, 3(a), and 3(d). Splitting the pulse into a pair of strongly overdetuned equal-area components with arbitrary power partition (we take $\widetilde{\Omega}=3.18$ and $W_{0} / W_{1}=17 / 3$ ) does not change the dynamics of collapse. This is seen from comparison of Figs. 3(a) and 3(d) with 3(b) and 3(e). In either case, the collapse stabilizes around $z$

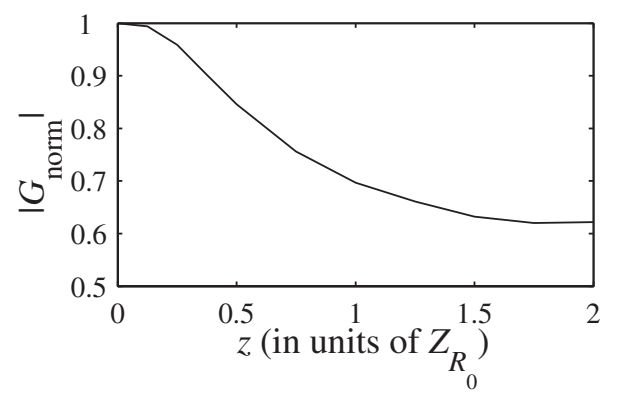

FIG. 4. Absolute value of the normalized coefficient of plasma wave-induced cross focusing, $G_{\text {norm }}=G_{N_{e}}^{\Omega}(z) / G_{N_{e}}^{\Omega}(0)$, versus propagation distance for the simulation of Figs. 3(c) and 3(f).

$\approx 2 Z_{R_{0}}$ by the formation of an evacuated electron density channel, $n_{e}(r=0)<0.1 n_{0}$ (not shown). The tightly focused pulses are further guided by the channel.

Next, we reduce the difference frequency to $\widetilde{\Omega}=1.25$ and demonstrate a total suppression of the RSF [yellow (light gray) curve in Fig. 2]. Intensity initially drops until $z$ $\approx 0.25 Z_{R_{0}}$. The inset in Fig. 2 shows perfect agreement with the bienvelope model. After this point, the PIC results depart from those of the bienvelope theory. Spectral analysis reveals one source of this disagreement. By analogy with Eqs. (1) and $(2 \mathrm{c})$ we define the plasma wave-induced cross-focusing coefficient in the frequency domain, $G_{N_{e}}^{\Omega}=N_{\omega}(\Omega) /$ $\left[a_{\omega}^{*}\left(\omega_{0}\right) a_{\omega}\left(\omega_{0}-\Omega\right)\right]$, where $a_{\omega}$ and $N_{\omega}$ are the Fourier transforms of the laser envelope and the density perturbation [Figs. 5(a) and 5(b)], and track its evolution along the laser path (Fig. 4). $G_{N_{e}}^{\Omega}$ is constant in the bienvelope model; hence, the defocusing persists all the way along the plasma (black curve in Fig. 2). Conversely, $G_{N_{e}}^{\Omega}$ gradually decays in the fully dynamic simulation, which turns the defocusing into a mild focusing. The collapse, however, does not occur: The laser spot size and longitudinal intensity profile change insignificantly over two Rayleigh lengths [Figs. 3(c) and 3(f)].

It should be noted that the electromagnetic cascading (EMC) [11-14] invalidates the bicolor approximation after $z \approx 0.25 Z_{R_{0}}$. Laser phase modulation by the plasma wave
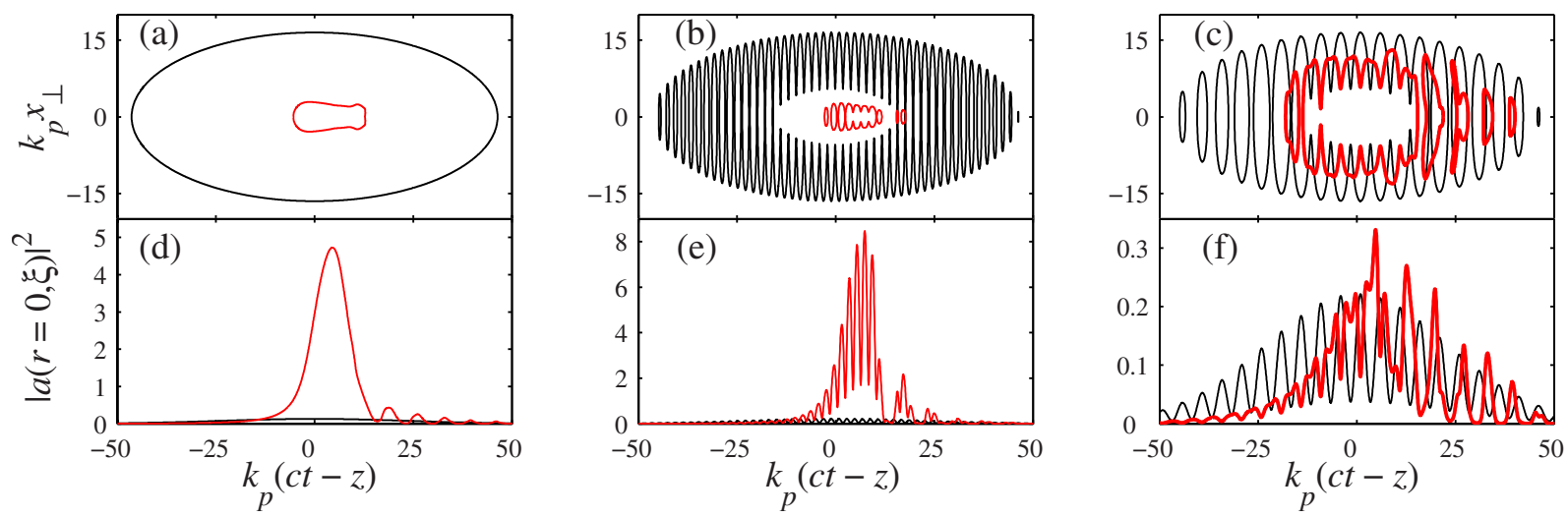

FIG. 3. (Color online) Radial and axial profiles of laser intensity taken at $z=0$ (black line) and $z=2 Z_{R_{0}}$ [red (dark gray) line]. Top: Isocontours at the level $\exp (-2)$ of the peak intensity. Bottom: Axial lineouts of normalized intensity. Left-hand column, initially monochromatic pulse with $W_{\text {tot }}=1.1$; middle column, bicolor pulse with the power partition $W_{0} / W_{1}=17 / 3, W_{0}+W_{1}=W_{\text {tot }}, \widetilde{\Omega}=3.18$; right-hand column, bicolor pulse with the same power partition and $\widetilde{\Omega}=1.25$. 

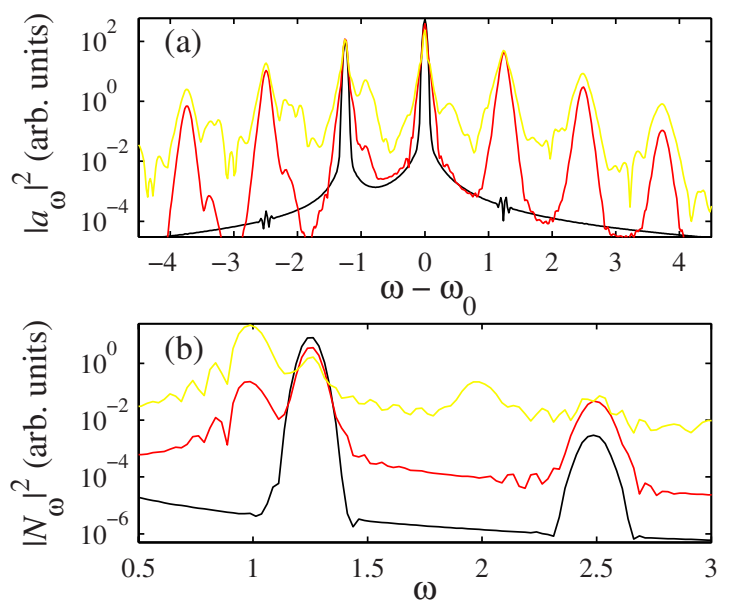

FIG. 5. (Color online) Spectral evolution of (a) laser radiation and (b) plasma density perturbations from the simulation of Figs. 3(c) and 3(f). Radially integrated spectra are shown for $z=0$ (black line), $z=Z_{R_{0}}$ [red (dark gray) line], $z=2 Z_{R_{0}}$ [yellow (light gray) line]. Frequencies are in units of $\omega_{p e}$.

with the amplitude (1) produces different spectral satellites shifted by integer multiples of $\Omega$ [Fig. 5(a)]. Requirement of no more than one satellite on either side of the original doublet is satisfied over the distance $z<Z_{R_{0}}\left[\left(\widetilde{\Omega}^{2}-1\right) /\right.$ $\left.\left(4 \widetilde{\Omega}^{2}\right)\right]\left(R W_{0} W_{1}\right)^{-1 / 2}$ [14]. For the parameters of Figs. 3(c) and 3(f), this is indeed one-quarter of a Rayleigh length. Including additional cascade sidebands into the multienvelope theory can improve the agreement with the fully dynamic PIC simulation.
The EMC saturates after $z \approx Z_{R_{0}}$. Nonresonant density perturbation $N_{\omega}(\Omega)$ that causes cascading gradually decays [see Fig. 5(b)]. The resonant plasma response $N_{\omega}\left(\omega_{p e}\right)$ becomes dominant, which is the signature of developing resonant self-modulation [5] and near-forward stimulated Raman scattering $[21,22]$. Indeed, the laser spectra in Fig. 5(a) include weak Raman features shifted by integer multiples of $\omega_{p e}$. Further evidence of the Raman instability is the erosion of the pulse tail clearly seen in the intensity profiles [Figs. 3(c) and 3(f)]. The total laser energy depletion is, however, very low $(<5 \%)$.

In conclusion, we have demonstrated that the relativistic self-focusing of a mildly overcritical long $\left(\omega_{p e} \tau_{L} \gg 1\right)$ laser pulse can be all-optically suppressed by splitting a small portion of its energy into a frequency-shifted (by $\Omega \gtrsim \omega_{p e}$ ) second pulse, and optically mixing the two in plasmas. The beat-wave-driven electron density perturbation (comoving periodic defocusing channel) entirely suppresses the RSF. This is validated by the fully relativistic 3D (with cylindrical symmetry) PIC simulations. With the RSF suppressed, the multicolor laser pulse is dynamically guided, which is the combined effect of beat-wave-driven electron density perturbations, electromagnetic cascading, and resonant selfmodulation. This guiding option is readily accessible for the experimental verification using state-of-the-art bicolor laser sources [23].

This work is supported by the U.S. Department of Energy under Contracts No. DE-FG02-04ER54763, No. DE-FG0204ER41321, No. DE-FG02-07ER54945, and by the NSF Grant No. PHY-0114336 administered by the FOCUS Center at the University of Michigan, Ann Arbor.
[1] Y. R. Shen, The Principles of Nonlinear Optics (Wiley, New York, 1984).

[2] A. G. Litvak, Sov. Phys. JETP 30, 344 (1969).

[3] C. E. Max, J. Arons, and A. B. Langdon, Phys. Rev. Lett. 33, 209 (1974).

[4] G. Z. Sun, E. Ott, Y. C. Lee, and P. Guzdar, Phys. Fluids 30, 526 (1987).

[5] E. Esarey, P. Sprangle, J. Krall, and A. Ting, IEEE J. Quantum Electron. 33, 1879 (1997).

[6] P. Mora and T. M. Antonsen, Jr., Phys. Plasmas 4, 217 (1997).

[7] B. Hafizi, A. Ting, P. Sprangle, and R. F. Hubbard, Phys. Rev. E 62, 4120 (2000).

[8] P. Sprangle, E. Esarey, and A. Ting, Phys. Rev. A 41, 4463 (1990).

[9] L. M. Gorbunov, S. Yu. Kalmykov, and P. Mora, Phys. Plasmas 12, 033101 (2005).

[10] S. E. Harris and A. V. Sokolov, Phys. Rev. Lett. 81, 2894 (1998).

[11] S. Yu. Kalmykov and G. Shvets, Phys. Rev. Lett. 94, 235001 (2005).

[12] P. Gibbon and A. R. Bell, Phys. Rev. Lett. 61, 1599 (1988);
61, 2509 (1988); 65, 1962 (1990); E. Esarey and A. Ting, ibid. 65, 1961 (1990).

[13] P. Gibbon, Phys. Fluids B 2, 2196 (1990).

[14] S. Yu. Kalmykov and G. Shvets, Phys. Plasmas 13, 056707 (2006).

[15] E. Esarey, A. Ting, and P. Sprangle, Appl. Phys. Lett. 53, 1266 (1988).

[16] C. J. McKinstrie and D. A. Russell, Phys. Rev. Lett. 61, 2929 (1988).

[17] C. V. Filip et al., Phys. Rev. E 69, 026404 (2004).

[18] G. Shvets and A. Pukhov, Phys. Rev. E 59, 1033 (1999).

[19] P. Sprangle, A. Ting, and C. M. Tang, Phys. Rev. A 36, 2773 (1987).

[20] R. Govil, W. P. Leemans, E. Y. Backhaus, and J. S. Wurtele, Phys. Rev. Lett. 83, 3202 (1999).

[21] T. M. Antonsen, Jr. and P. Mora, Phys. Fluids B 5, 1440 (1993).

[22] K.-C. Tzeng and W. B. Mori, Phys. Rev. Lett. 81, 104 (1998).

[23] F. Grigsby, Dong Peng, and M. C. Downer, J. Opt. Soc. Am. B 25, 346 (2008). 